\title{
Prevalencia de diabetes mellitus e hiperlipidemias en indígenas otomíes
}

\author{
Claudia A lvarado-0 suna, Q FB, ${ }^{(1)}$ Feliciano Milian-Suazo, MVZ, MSc, PhD, ${ }^{(1)}$ \\ Victoria Valles-Sánchez, D r en C. (2)
}

Alvarado-Osuna C, Milian-Suazo F,Valles-SánchezV. Prevalencia de diabetes mellitus e hiperlipidemias en indígenas otomíes. Salud Publica Mex 2001;43:459-463. El texto completo en inglés de este artículo está disponible en: http://www.insp.mx/salud/index.html

\section{Resumen}

Objetivo. Determinar prevalencia y factores de riesgo de la diabetes mellitus (DM) e hiperlipidemias en un grupo de indígenas otomíes de Q uerétaro. Material y métodos. Entre 1996 y 1997, en muestreo de conveniencia se trabajó con 91 indígenas, de 15 a 77 años de edad, de las comunidades de Yosphí y El Rincón, del estado de Q uerétaro, México. Se tomaron muestras sanguíneas en ayuno y se determinó la concentración de glucosa, colesterol y triglicéridos. Se realizó análisis estadístico para comparación entre sexos y grupos de edad. Resultados. La prevalencia de DM fue $4.4 \%$, la de hipercolesterolemia $7.2 \%$, y la de hipertrigliceridemia (HTG) 26\%. Las concentraciones promedio de glucosa $(81.0 \pm 24.4 \mathrm{mg} / \mathrm{dl})$ y triglicéridos $(157.4 \pm 88.9 \mathrm{mg} / \mathrm{dl}) \mathrm{se}$ incrementaron significativamente con la edad ( $p=0.0279$ y $p<0.0001$ respectivamente), de igual manera para la prevalencia de HTG $(p<0.0001)$. Conclusiones Los resultados sugieren que cambios drásticos en los patrones tradicionales de alimentación que conservan los indígenas otomíes pueden originar problemas de salud asociados a la elevación de lípidos en sangre. El texto completo en inglés de este artículo está disponible en: http://www.insp.mx/salud/index.html

Palabras clave: indígenas mexicanos; diabetes mellitus; hipercolesterolemia; hipertrigliceridemia; prevalencia; México

\author{
Alvarado-Osuna C, Milian-Suazo F, Valles-Sánchez V. \\ Prevalence of diabetes mellitus \\ and hyperlipemias in Otomi indians. \\ Salud Publica Mex 2001:43:459-463. \\ The English version of this paper \\ is available at: http://www.insp.mx/salud/index.html
}

\begin{abstract}
A bstract
Objective. To determine prevalence and risk factors for diabetes mellitus (DM) and hyperlipidemias in a population of 0 tomi Indians. Material and methods A cross-sectional study was conducted between 1996 and 1997, in a convenience sample of 910 to mi Indians, aged 15 to 77 years, in the comunities of Yosphi and El Rincon, Q ueretaro, Mexico. Fasting blood samples were obtained to measure glucose, cholesterol and triglyceride levels. Results. DM was found in $4.4 \%$ of subjects; hypercholesterolemia in $7.6 \%$; and hypertriglyceridemia (HTG) in 26\%. Mean concentrations of glucose $-(81.0 \pm 24.4 \mathrm{mg} / \mathrm{dl})$ and triglycerides $(157.4 \pm 88.9$ $\mathrm{mg} / \mathrm{dl}$ ) increased significantly with age, $p=0.0279$ and $p<0.0001$, respectively; as well as the prevalence of HTG $(p<0.0001)$. Conclusions. Results suggest that drastic changes in the diet of 0 tomi Indians may cause severe problems related to high concentration of lipids in blood. The English version of this paper is available at: http:// www.insp.mx/salud/index.html
\end{abstract}

Key words: Mexican indians; diabetes mellitus; hypercholesterolemia; hypertriglyceridemia; prevalence; Mexico

(1) Licenciatura en N utrición, Universidad Autónoma de Q uerétaro.

(2) Departamento de Endocrinología, Instituto $\mathrm{N}$ acional de Ciencias Médicas y N utrición Salvador Zubirán.

Fecha de recibido: 19 de junio de 2000 - Fecha de aprobado: 30 de julio de 2001

Solicitud de sobretiros: Q FB Claudia Alvarado 0 suna. Hacienda Escolásticas N 0. 307. Jardines de la Hacienda 76180 Q uerétaro, Q ro. Correo electrónico: anai@ sunserver.uaq.mx 
a diabetes mellitus (DM) era una enfermedad rara $L$ en el nuevo mundo hasta principios del siglo XX. A partir de 1940 empezaron a reportarse casos de diabetes en las comunidades indígenas de Norteamérica y en la actualidad se ha informado de comunidades Pimas donde $50 \%$ de la población mayor de 35 años padece la enfermedad. ${ }^{1-3}$ Desde entonces se ha postulado que las poblaciones indoamericanas poseen un gen ahorrador ${ }^{4}$ que las hace susceptibles a DM, obesidad y dislipidemias cuando cambian sus patrones de alimentación.

En México la DM ha tenido un ascenso alarmante, en 1970 la diabetes como causa de muerte ocupó la posición número 12 , actualmente se encuentra en la tercera posición. 5,6 Por otra parte, la Encuesta Nacional de Enfermedades Crónicas (ENEC) ${ }^{7}$ ha reportado la prevalencia de hipercolesterolemia $(\mathrm{HC})$ en $8.8 \%$ y de hipertrigliceridemia (HTG) de 5.4\%.

En México se han realizado pocos estudios sobre prevalencia de trastornos metabólicos en grupos indígenas. Se reportaron prevalencias de DM 6.3\% y $10.5 \%$ en hombres y mujeres, respectivamente, de tribus $\mathrm{Pi}$ mas de Sonora, ${ }^{8} 2.1 \%$ en Mazatecas de Oaxaca ${ }^{9}$ y no se observaron casos de diabetes en poblaciones Tepehuana, Huichol y Mexicanera en Durango. ${ }^{10}$

En el estado de Querétaro existen núcleos de indígenas otomíes reconocidos oficialmente. Dentro de éstos se encuentran Yosphí y El Rincón, en el municipio de Amealco, que han conservado rasgos étnicos tales como la lengua, el vestido y la organización familiar, ${ }_{11}^{11}$ así como rasgos genéticos propios de grupos indoamericanos. ${ }^{12-14}$

El estudio de enfermedades como la DM y las hiperlipidemias en los indígenas mexicanos aporta información sobre los factores ambientales que inciden, con respecto a poblaciones genéticamente similares, pero con estilo de vida diferentes, como son los indígenas norteamericanos.

\section{Material y métodos}

Entre noviembre de 1996 y febrero de 1997 se estudió a un grupo de 91 individuos (73 mujeres y 18 hombres) otomíes, aparentemente sanos, de 15 a 77 años de edad, habitantes de las comunidades de Yosphí y El Rincón, en el estado de Querétaro, y que aceptaron participar en la investigación. Se les tomó una muestra de $3 \mathrm{ml}$ de sangre por punción venosa y luego de un ayuno mínimo de 12 horas. Se efectuaron determinaciones de glucosa, colesterol y triglicéridos mediante métodos enzimáticos convencionales. A cada sujeto se le aplicó un cuestionario donde se registraron los alimentos con- sumidos en las últimas 24 horas (recordatorio de 24 horas).

El tamaño de muestra para estimar la prevalencia poblaciona ${ }^{15}$ se determinó considerando una prevalencia del 5\% para DM, y un margen de error de cuatro puntos porcentuales, con un nivel de confianza del 95\%. El mínimo de individuos a estudiar fue de 114, de los cuales se logró obtener a 91 voluntarios. La DM se definió como una glucemia en ayuno $\geq 126 \mathrm{mg} / \mathrm{dl}$, intolerante a la glucosa (IG) como una glucemia de entre 110 a $125 \mathrm{mg} / \mathrm{dl} .{ }^{16}$ La hipercolesterolemia se definió para valores de colesterol en suero $>240 \mathrm{mg}$ / dl. ${ }^{17}$ Se consideró HTG a valores de triglicéridos en suero superiores a los $200 \mathrm{mg} / \mathrm{dl}$.

Se obtuvieron prevalencias crudas y estandarizadas por sexo y edad, categorizando esta última en cinco grupos etáreos. Se analizaron diferencias entre las medias de las variables continuas para sexo por medio de la prueba $t$ de Student, y para grupos etáreos, por medio de análisis de varianza (ANOVA). La comparación de medias a posteriori se hizo por medio de la prueba de Duncan utilizando un alfa de 0.05. La asociación entre las prevalencias de las enfermedades por edad y sexo se hizo mediante análisis de ji-cuadrada para tendencias. Todos los análisis estadísticos se llevaron a cabo con el paquete computacional SAS. ${ }^{18}$

La información obtenida en la entrevista sobre alimentación se analizó a manera de dieta habitual mediante el paquete computacional NutriPac. ${ }^{19}$

\section{Resultados}

La prevalencia de DM fue de 4.4\% (IC95\% 0.1-8.7) al igual que la IG. La prevalencia de HC fue del 7.2\% (IC95\% 1.5-12.9), y la de HTG de 26.0\% (IC95\% 15.7-36.3).

La concentración promedio de glucosa se incrementó significativamente con la edad $(p=0.0279)$; dicha diferencia se observó en los grupos extremos de edades. Los triglicéridos mostraron una elevación significativa después de los 40 años $(p<0.0001)$. Se observó un incremento de la prevalencia de DM o IG con respecto a la edad, aunque dicho incremento no fue significativo $(p=0.1522)$. La prevalencia de HTG se incrementó significativamente con la edad $(p<0.0001)$, cuadro I.

El género del individuo no intervino de manera significativa en ninguna variable, con excepción de la prevalencia de DM e IG ( $p=0.0225$ ); las mujeres exhibieron una mayor tendencia de presentar IG mientras que los hombres presentaron mayor prevalencia de DM, cuadro II. 


\section{Cuadro I

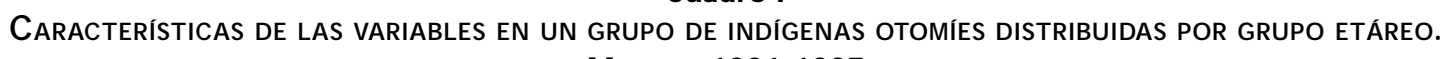 Méxıco, 1991-1997}

\begin{tabular}{|c|c|c|c|c|c|c|}
\hline \multirow[b]{2}{*}{ Variables } & \multicolumn{5}{|c|}{ Edad } & \multirow[b]{2}{*}{$p$} \\
\hline & $15-20$ & $21-30$ & $31-40$ & $41-50$ & $>50$ & \\
\hline$n(\%)$ & $11(12.1)$ & $46(50.5)$ & 13(14.3) & 14(15.4) & $7(7.7)$ & \\
\hline Glucosa (mg/dl)* & $69.4 \pm 14.8^{\mathrm{a}}$ & $77.2 \pm 23.6^{\mathrm{ab}}$ & $81.9 \pm 16.0^{a b}$ & $96.8 \pm 24.6^{b}$ & $90.6 \pm 38.6^{b}$ & 0.0279 \\
\hline Colesterol (mg/dl)p & $154.7 \pm 34.5$ & $177.3 \pm 46.7$ & $166.9 \pm 36.6$ & $166.2 \pm 37.2$ & $198.0 \pm 38.2$ & 0.2671 \\
\hline Triglicéridos(mg/dl)*g & $93.4 \pm 49.9^{a}$ & $138.7 \pm 77.3^{\mathrm{a}}$ & $133.6 \pm 41.9^{a}$ & $234.9 \pm 84.8^{b}$ & $280.0 \pm 94.4^{b}$ & $<0.0001$ \\
\hline \multicolumn{7}{|c|}{ Prevalencia de DM o Intolerancia a la glucosa \% } \\
\hline Normal & 12.1 & 46.1 & 14.3 & 12.1 & 6.6 & 0.1522 \\
\hline DM o Intolerancia & 0.0 & 4.4 & 0.0 & 3.3 & 1.1 & \\
\hline \multicolumn{7}{|c|}{ Prevalencia hipercolesterolemia $p^{\ddagger}$} \\
\hline Normal & 12.0 & 44.6 & 13.3 & 14.5 & 8.4 & 0.0803 \\
\hline Hipercolesterolemia & 0.0 & 7.2 & 0.0 & 0.0 & 0.0 & \\
\hline \multicolumn{7}{|c|}{ Prevalencia hipertriglicéridemia g $g^{\S}$} \\
\hline Normal & 11.0 & 45.2 & 11.0 & 5.4 & 1.3 & $<0.0001$ \\
\hline Hipertrigliceridemia & 0.0 & 11.0 & 0.0 & 9.6 & 5.5 & \\
\hline \multicolumn{7}{|c|}{$\begin{array}{l}\text { Los datos se muestran como media } \pm \text { desviación estándar para variables continuas y prevalencia para variables nominales } \\
* \text { Medias con la misma letra son significativamente iguales con la prueba de Duncan a posteriori con alfa }=0.05 \\
\text { } \text { Se cuenta con } 83 \text { medidas } \\
\text { \$ Se cuenta con } 73 \text { medidas }\end{array}$} \\
\hline
\end{tabular}

Cuadro II

\section{Características de las variables en Un GRUPo de INDÍGENAS OTOMÍES DISTRIBUIDAS POR SEXO. MÉxICO, 1991-1997}

\begin{tabular}{|c|c|c|c|c|}
\hline \multirow[b]{2}{*}{ Variables } & \multicolumn{2}{|c|}{ Sexo } & \multirow[b]{2}{*}{ Total } & \multirow[b]{2}{*}{$p^{*}$} \\
\hline & Femenino & Masculino & & \\
\hline$n(\%)$ & $73(80.2)$ & 18(19.8) & $91(100)$ & \\
\hline Glucosa (mg/dl) & $79.9 \pm 20.9$ & $85.3 \pm 35.7$ & $81.0 \pm 24.4$ & 0.4055 \\
\hline Colesterol (mg/dl)p & $174.8 \pm 43.4$ & $167.7 \pm 40.2$ & $173.4 \pm 42.6$ & 0.5427 \\
\hline Triglicéridos(mg/dl)g & $152.9+81.9$ & $173.3 \pm 112.2$ & $157.4 \pm 88.9$ & 0.4222 \\
\hline \multicolumn{5}{|c|}{ Prevalencia de DM o Intolerancia a la glucosa \% } \\
\hline Normal & 74.7 & 16.5 & 91.2 & 0.0225 \\
\hline Intolerancia & 4.4 & 0.0 & 4.4 & \\
\hline DM & 1.10 & 3.3 & 4.4 & \\
\hline
\end{tabular}

Prevalencia hipercolesterolemia $\mathrm{p}^{\ddagger}$

\begin{tabular}{lrrrr} 
N ormal & 73.5 & 19.3 & 92.8 & 0.7593 \\
\hline Hipercolesterolemia & 6.0 & 1.2 & 7.2 &
\end{tabular}

Prevalencia hipertrigliceridemia $\mathrm{g}^{\S}$

\begin{tabular}{lrrrr} 
N ormal & 57.5 & 16.4 & 74.0 & 0.6558 \\
\hline Hipertrigliceridemia & 20.6 & 5.5 & 26.0 &
\end{tabular}

* Comparación entre sexos

₹ Se cuenta con 83 medidas

\& Se cuenta con 73 medidas
Los alimentos mencionados con mayor frecuencia en el recordatorio de 24 horas fueron: tortilla (100.0\%), frijol (87.0\%), chile (71\%), nopal (37.7\%), café $(27.3 \%)$, huevo $(15.6 \%)$, quelite $(13.0 \%)$, arroz $(11.7 \%)$, calabaza $(10.4 \%)$, y sopa de pasta $(10.4 \%)$. La dieta habitual en las comunidades otomíes es abundante en hidratos de carbono complejos, fibra dietética y baja en proteína de origen animal y grasas saturadas.

\section{Discusión}

La prevalencia de DM observada en este estudio (4.4\%) concuerda con las bajas prevalencias reportadas en otras poblaciones indígenas mexicanas $\left(0.0 \%{ }^{10} \mathrm{y}\right.$ $2.01 \%$ ). ${ }^{9}$ Dichas prevalencias han resultado consistentemente inferiores a las observadas tanto en poblaciones indígenas norteamericanas ${ }^{2,3}$ como en poblaciones urbanas mestizas mexicanas. ${ }^{7}$

La prevalencia de DM observada es casi la mitad de lo informado en el país, de $8.2 \%, 7$ considerando que nuestro tamaño de muestra $(n=91)$ es mucho más pequeño que el de la ENEC. Cabe mencionar que en este estudio se definió la DM bajo criterios de la ADA, ${ }^{16}$ mientras que lo reportado hasta 1998 se analizó bajo criterios de la $\mathrm{OMS}^{20}$ (DM glucemia de ayuno $\geq 140$ 
$\mathrm{mg} / \mathrm{dl}$ ). Calculando la prevalencia de DM del presente estudio con criterios de la OMS se observa que éste es del $3.3 \%$.

La mayoría de los diabéticos $(75 \%)$ fueron hombres a pesar de que están subrepresentados en la muestra; esto difiere a lo reportado para pimas ${ }^{8}$ en estudios con características similares y en donde se observó sólo el 33\% de diabéticos hombres. Esto puede atribuirse a la estrategia de vida que siguen los indígenas del bajío, ${ }^{21}$ en donde los hombres salen de sus comunidades a buscar trabajo a la ciudad, cambiando por consecuencia sus hábitos alimentarios. Este hecho pone de manifiesto la enorme susceptibilidad de los grupos indígenas a la alteración de su estilo de vida.

La prevalencia de HC encontrada (7.2\%) y la concentración promedio de colesterol $(173.4 \mathrm{mg} / \mathrm{dl})$ son similares a lo informado nacionalmente en población urbana $(8.8 \%$ y $182 \mathrm{mg} / \mathrm{dl}){ }^{7}$ considerando nuevamente las diferencias en los tamaños de muestra. Este hecho llama la atención, ya que los indígenas tienen consumos de productos de origen animal muy bajos. Estudios hechos a indígenas tarahumaras reportan colesterolemias promedio de $136 \mathrm{mg} / \mathrm{dl}_{1}{ }^{22} 30 \%$ inferiores a las concentraciones en los otomíes con patrones alimentarios similares.

La prevalencia de HTG en los otomíes (26.1\%) fue superior a la nacional $(20.8 \%$ para un valor de corte de $200 \mathrm{mg} / \mathrm{dl}) .{ }^{7}$ La concentración promedio de triglicéridos en otomíes ( $157.4 \mathrm{mg} / \mathrm{dl}$ ) fue un $36 \%$ inferior al promedio nacional $(214 \mathrm{mg} / \mathrm{dl}) .^{7}$ Los otomíes no presentaron triglicéridos superiores a $400 \mathrm{mg} / \mathrm{dl}$, a diferencia de la población urbana. ${ }^{7}$ Por otra parte, las concentraciones de triglicéridos se incrementan notablemente después de los 40 años $(p<0.0001)$, patrón que difiere de lo reportado para pimas norteamericanos, ${ }^{23}$ quienes muestran descenso de la trigliceridemia después de los 45 años.

Los triglicéridos sanguíneos de la población mexicana se han reconocido más elevados que entre la población norteamericana. ${ }^{24}$ Dichas diferencias se han atribuido a la dieta elevada en hidratos de carbono de aquélla ${ }^{25}$ efecto que se amplifica en la dieta indígena cuya principal fuente de energía son las tortillas y las leguminosas. Si bien existe controversia sobre el impacto de la HTG en la salud, actualmente se considera que existe una asociación positiva entre la HTG y riesgo cardiovascular. ${ }^{26}$

Por otra parte, estudios en tarahumaras han mostrado que la introducción de dietas ricas en grasas saturadas originan elevación en los lípidos sanguíneos. ${ }^{27}$

Se sabe que existen diferencias metabólicas de origen genético que hacen a los indoamericanos espe- cialmente susceptibles a enfermedades crónico-degenerativas. ${ }^{28}$ Las evidencias sugieren que la dieta ha jugado hasta la fecha un papel protector en los indígenas mexicanos. Del presente trabajo se hace evidente la necesidad de un estudio que precise los factores de la dieta implicados en el fenómeno de la nutrición de grupos indígenas, que apoye el diseño de medidas preventivas ante la inminente transculturación de los tradicionales patrones de alimentación en las comunidades indígenas mexicanas.

\section{Referencias}

1. West K. Diabetes in American indians and other native populations of the N ew W orld. Diabetes 1974;23:841-854.

2. Bennett P, Burch T, Miller M. Diabetes mellitus in American (Pima) indians. Lancet 1971:125-128.

3. G ohdes D, Kaufman S,Valway S. D iabetes in American indians. Diabetes Care 1993, 16S1:239-243.

4. N eel J. Diabetes mellitus:A "thrifty" genotype rendered detrimental by "progress"? Am J Hum G enet 1962; 14:353-362.

5. Chávez A, Muñoz M, Roldán J, Bermejo S,AvilaA. La nutrición en México y la transición epidemiológica. México, D.F.: Instituto $\mathrm{N}$ acional de la N utrición Salvador Zubirán, 1993.

6. Mortalidad 1997. Dirección general de estadística e informática. México, D.F.: Secretaría de Salud, 1998.

7. Encuesta Nacional de Enfermedades Crónicas 1993. Dirección General de Epidemiología, Instituto $\mathrm{N}$ acional de la N utrición. México, D.F.: Secretaría de Salud, 1993.

8. Ravussin E,Valencia M, Esparza J, Bennett P, Schulz L. Effects of a traditional lifestyle on obesitiy in Pima indians. Diabetes C are 1994, 17:10671073.

9. Castro S, Escobedo P. La prevalencia de la diabetes mellitus no dependiente de insulina y factores de riesgo asociados, en población mazateca del estado de 0 axaca, México. Gac Med Mex 1997;133:527-534.

10. Guerrero-Romero F, Rodríguez M, Sandoval F. Low prevalence of noninsulin-dependent diabetes mellitus in indigenous communities of Durango, Mexico.Arch Med Res 1997;28:137-140.

11. Embriz A. Q uerétaro, Indicadores socioeconómicos de los pueblos indígenas de México por centro coordinador indigenista, lengua principal y localidades eminentemente indígenas. México, D.F.: Instituto $\mathrm{N}$ acional Indigenista, 1994.

12. Alvarado 0, Barrera A, Terán L, C amarena 0, Juárez A. Frecuencias de antígenos eritrocitarios y leucocitarios en indígenas otomíes de $\mathrm{Q}$ uerétaro. Resúmenes del segundo coloquio 0 topame. México,D.F.:MN A, 1998. 13. Lisker R. Estructura genética de la población mexicana, aspectos médicos y antropológicos. México, D.F.: Salvat Editores, 1981.

14. Gorodezky C.Variación genética del MHC en la población mexicana. Histocomp Lat Amer 1988;1:8-12.

15. D anielW . Bioestadística, base para el análisis de las ciencias de la salud. Ed. México D.F.: UTEHA N oriega Editores, 1998.

16. Report of the Experte Committee on the Diagnosis and classification of Diabetes Mellitus. Diabetes Care 1997;20:1183-1195.

17. $\mathrm{N}$ ational Institutes of Health. Recommendations for Improving $\mathrm{Cho-}$ lesterol Measurement. Bethesda (MA): N IH, 1990.

18. SAS Institute Inc. SAS/STAT guía de usuario, versión 6.11. C ary, N orth Caroline: SA S Institute, 1995. 
19. Ledesma J, Hernández $\mathrm{S}, \mathrm{C}$ haparo $\mathrm{A}$. N utriPac, programa computacional para N utrición, versión 1.5, 1992-1993.

20.W HO Expert committee on diabetes mellitus. G inebra:W orld Health O rganization, 1980

21. Serna A. La migración en la estrategia de la vida rural. Q uerétaro: Universidad Autónoma de Q uerétaro, 1996.

22. Connor W, Cerqueira M, Connor R, W allace R, Malinow M, Casdorph $H$. The plasma lipids, lipoproteins, and diet of the Tarahumara indians of Mexico.Am J Clin N utr 1978;31:1131-1142.

23. Howard B, Davis M, Pettitt D, Knowler W, Bennett P. Plasma and lipoprotein cholesterol and trigliceride concentrations in the Pima indians: Distributions differing fron those of Caucasians. Circulation 1983;68: 714-724.

24. Stern M, González C, Mitchell B, Villalpando E, Haffner S, Hazuda H. Genetic and environmental determinants of type II diabetes in Mexico city and San Antonio. Diabetes 1992:41;484-492.
25. Parks E, Hellerstein M. Carbohydrate-induced hypertiacylglycerolemia: Historical perspective and review of biological mechanisms. Am J Clin N utr 2000;71:412-433.

26. Gotto A.Triglyceride as a risk factor for coronary artery disease.Am J Cardiol 1998:82:22-25.

27. McMurry M, Cerqueira M, Connor S, Connor W. Changes in lipid and lipoprotein levels and body weight in Tarahumara indians after consumption of an affluen diet. N Engl J Med 1991:325;1704-1708.

28. Howard B, Bogardus C, Ravussin E, Foley J, Lillioja S, Mott D et al. Studies of the etiology of obesity in Pima indians. Am J Clin Nutr 1991; 53:1577S-1585S 